\title{
$U(n)$-INVARIANT KÄHLER METRICS WITH NONNEGATIVE QUADRATIC BISECTIONAL CURVATURE*
}

\author{
SHAOCHUANG HUANG ${ }^{\dagger}$ AND LUEN-FAI TAM TA $^{\ddagger}$
}

\begin{abstract}
By perturbing the complete $U(n)$-invariant metrics with positive bisectional curvature constructed by Wu-Zheng [10], we obtain complete $U(n)$-invariant Kähler metrics on $\mathbb{C}^{n}$, $n \geq 3$, which have nonnegative quadratic bisectional curvature (QB $\geq 0$ ) everywhere, and which do not have nonnegative orthogonal bisectional curvature and do not have nonnegative Ricci curvature at some points. We prove that $\mathrm{QB} \geq 0$ is preserved under the Kähler-Ricci flow for complete $U(n)$-invariant solution with bounded curvature. We prove that Ric $\geq 0$ is also preserved under an additional assumption.
\end{abstract}

Key words. $U(n)$-invariant Kähler metrics, quadratic bisectional curvature, Kähler-Ricci flow.

AMS subject classifications. Primary 32Q15; Secondary 53C44.

1. Introduction. Let $\left(M^{n}, g\right)$ be a Kähler manifold of complex dimension $n$ and let $o \in M . M$ is said to have nonnegative bisectional curvature (abbreviated as $\mathbf{B} \geq 0$ ) at $o$ if for any $X, Y \in T_{o}^{(1,0)}(M), R(X, \bar{X}, Y, \bar{Y}) \geq 0 . M$ is said to have nonnegative orthogonal bisectional curvature (abbreviated as $\mathbf{B}^{\perp} \geq 0$ ) at $o$ if $R(X, \bar{X}, Y, \bar{Y}) \geq 0$ for all unitary pairs $X, Y \in T_{o}^{(1,0)}(M) . \quad M$ is said to have nonnegative quadratic orthogonal bisectional curvature at $o$ (abbreviated as $\mathbf{Q B} \geq 0$ ) if for any unitary frame $e_{i}$ at $o$ and real numbers $\xi_{i}$ we have

$$
\sum_{i, j} R_{i \bar{i} j \bar{j}}\left(\xi^{i}-\xi^{j}\right)^{2} \geq 0
$$

Here $R_{i \bar{i} j \bar{j}}=R\left(e_{i}, \bar{e}_{i}, e_{j}, \bar{e}_{j}\right) . \mathbf{B}>0$ and $\mathbf{B}^{\perp}>0$ are defined similarly. It is obvious that $\mathbf{B} \geq 0 \Rightarrow \mathbf{B}^{\perp} \geq 0 \Rightarrow \mathbf{Q B} \geq 0$. Note that in dimension $n=2$, the conditions $\mathbf{B}^{\perp} \geq 0$ and $\mathbf{Q B} \geq 0$ are the same. We will say that $M$ satisfies $\mathbf{B} \geq 0$ (respectively $\mathbf{B}^{\perp} \geq 0, \mathbf{Q B} \geq 0$ ) provided that $M$ has $\mathbf{B} \geq 0$ (respectively $\mathbf{B}^{\perp} \geq 0, \mathbf{Q B} \geq 0$ ) at every point.

Even though the condition $\mathbf{Q B} \geq 0$ is weaker than the condition that bisectional curvature is nonnegative, Kähler manifolds with $\mathbf{Q B} \geq 0$ still have many interesting properties. In fact the condition $\mathbf{Q B} \geq 0$ appeared implicitly in [3] and it was proved that a real harmonic $(1,1)$ form on a compact Kähler manifold with this curvature condition must be parallel. In [9] (see also [11, 1]), it was proved on a compact Kähler manifold with $\mathbf{Q B} \geq 0$ any numerically effective (nef) line bundle admits a smooth metric with nonnegative curvature. In [1], it was proved that any Kähler manifold with $\mathbf{Q B} \geq 0$ must have nonnegative scalar curvature and must be flat if the scalar curvature is zero and if the manifold has complex dimension at least three. It was also proved that an irreducible compact Kähler manifold with $\mathbf{Q B} \geq 0$ has positive first Chern class. It is then a question whether there are Kähler manifolds satisfying $\mathbf{Q B} \geq 0$ but not $\mathbf{B} \geq 0$ or even $\mathbf{B}^{\perp} \geq 0$. In [4] Li-Wu-Zheng are able to construct the

\footnotetext{
${ }^{*}$ Received February 5, 2013; accepted for publication September 11, 2013.

$\dagger$ The Institute of Mathematical Sciences and Department of Mathematics, The Chinese University of Hong Kong, Shatin, Hong Kong, China (schuang@math.cuhk.edu.hk).

$\ddagger$ The Institute of Mathematical Sciences and Department of Mathematics, The Chinese University of Hong Kong, Shatin, Hong Kong, China (lftam@math.cuhk.edu.hk). Research partially supported by Hong Kong RGC General Research Fund \#CUHK 403011.
} 
first example of compact Kähler manifold which satisfies $\mathbf{Q B} \geq 0$ but not $\mathbf{B}^{\perp} \geq 0$. The example is a Kähler $C$-space. In [2], necessary and sufficient conditions on any Kähler $C$-space with $b_{2}=1$ satisfies $\mathbf{Q B} \geq 0$ are obtained by Chau and the second author. In particular, many more examples of compact Kähler manifolds satisfying QB $\geq 0$ but not $\mathbf{B}^{\perp} \geq 0$ are given.

There is a problem on complete noncompact Kähler manifold on solving the Poincaré-Lelong equation. Namely, given a closed real $(1,1)$ form $\rho$, one would like to find a potential function $u$ so that $\sqrt{-1} \partial \bar{\partial} u=\rho$. Previous works assume that the bisectional curvature is nonnegative and decay suitably, see references in [6]. However, the result is still true if we only assume $\mathbf{Q B} \geq 0$ and Ric $\geq 0$. So it is also interesting to see if there are noncompact examples with $\mathbf{Q B} \geq 0$ but not $\mathbf{B}^{\perp} \geq 0$.

There are not very many examples of complete noncompact Kähler manifolds with nonnegative bisectional curvature. In [10] $\mathrm{H}$. Wu and Zheng systematically studied $U(n)$-invariant Kähler metrics on $\mathbb{C}^{n}$ with positive bisectional curvature and are able to give many examples. In this paper, by perturbing a metric constructed in [10] we are going to construct examples of complete noncompact Kähler manifolds which satisfy $\mathbf{Q B} \geq 0$ but not $\mathbf{B}^{\perp} \geq 0$. Each such metric with positive bisectional curvature in [10] is determined up to scaling by a smooth function $\xi$ on $[0, \infty)$ such that $\xi(0)=0$, $0<\xi<\infty$ on $(0, \infty)$ and $\xi^{\prime}>0$. We will show that given such a $\xi$, one can find $R>1, \bar{\xi}$ such that $\bar{\xi}=\xi$ outside $[R-1, R+1]$ and $0<\bar{\xi}<1$ on $(0, \infty)$, such that the $U(n)$-invariant Kähler metric on $\mathbb{C}^{n}(n \geq 3)$ determined by $\bar{\xi}$ satisfies QB $\geq 0$ but not $\mathbf{B}^{\perp} \geq 0$. See Theorem 4.1 for more details. Actually, the metrics also do not satisfy Ric $\geq 0$.

Motivated by the generalized Hartshorne conjecture, see [12, p.218], Li-Wu-Zheng [4] conjectured that very irreducible compact Kähler manifold with $\mathbf{Q B}^{\perp} \geq 0$ must be biholomorphic to a Kähler $C$-space. It is natural to see if one might use KählerRicci flow. However, it is unclear if the condition $\mathbf{Q B} \geq 0$ is preserved under the Kähler-Ricci flow. In this work, we will prove that this curvature condition is preserved for $U(n)$-invariant solution of the Kähler-Ricci flow provided the curvature is bounded. With an additional condition, we will prove that the condition Ric $\geq 0$ is also preserved.

The paper is organized as follows. In $\S 2$, we will review the construction of $\mathrm{H}$. $\mathrm{Wu}$ and Zheng [10] on $U(n)$-invariant Kähler metrics on $\mathbb{C}^{n}$. In $\S 3$, we will give characterization of $\mathbf{B}^{\perp} \geq 0$ and $\mathbf{Q B} \geq 0$ for $U(n)$-invariant Kähler metrics. In $\S 4$, we will construct the examples mentioned above. In $\S 5$, we will discuss preservation of some curvature conditions including $\mathbf{Q B} \geq 0$ and Ric $\geq 0$ for the $U(n)$-invariant solution of the Kähler-Ricci flow.

2. The construction of Wu-Zheng. In this work, we always assume that $n \geq 3$. We first recall the construction of $U(n)$-invariant Kähler metrics on $\mathbb{C}^{n}$ by $\mathrm{H}$. Wu and Zheng [10]. In the standard coordinates of $\mathbb{C}^{n}$, the general form of an $U(n)$-invariant Kähler metric on $\mathbb{C}^{n}$ is given by:

$$
g_{i \bar{j}}=f(r) \delta_{i j}+f^{\prime}(r) \bar{z}_{i} z_{j}
$$

where $r=|z|^{2}, f$ is a smooth function on $[0, \infty)$. Let $h=(r f)^{\prime}$. The following is in [10].

LEMMA 2.1. $g$ is complete Kähler metric if and only if $f>0, h>0$ and

$$
\int_{0}^{\infty} \frac{\sqrt{h}}{\sqrt{r}} d r=\infty .
$$


If $h>0$, then $\xi=-\frac{r h^{\prime}}{h}$ is a smooth function on $[0, \infty)$ with $\xi(0)=0$. On the other hand, if $\xi$ is a smooth positive function on $[0, \infty)$ with $\xi(0)=0$. Define $h(r)=h(0) \exp \left(-\int_{0}^{r} \frac{\xi(s)}{s} d s\right)$ and $f(r)=\frac{1}{r} \int_{0}^{r} h(s) d s$ with $h(0)>0$. Then (2.1) define a $U(n)$-invariant Kähler metric on $\mathbb{C}^{n}$. The following is also from [10].

THEOREM 2.1.

(i) If $0<\xi<1$ on $(0, \infty)$, then $g$ is complete.

(ii) $g$ is complete and has positive bisectional curvature if and only if $\xi^{\prime}>0$ and $0<\xi<1$ on $(0, \infty)$.

(iii) Every complete $U(n)$-invariant Kähler metric on $\mathbb{C}^{n}$ with positive bisectional curvature is given by a smooth function $\xi$ in (ii).

The curvature tensor of a $U(n)$-invariant Kähler metric under the unitary frame

$$
\left\{e_{1}=\frac{1}{\sqrt{h}} \partial_{z_{1}}, e_{2}=\frac{1}{\sqrt{f}} \partial_{z_{2}}, \cdots, e_{n}=\frac{1}{\sqrt{f}} \partial_{z_{n}}\right\}
$$

at the point $z=\left(z_{1}, 0, \cdots, 0\right)$ is given as follows, see [10]:

$$
\begin{aligned}
& A:=R_{1 \overline{1} 1 \overline{1}}=\frac{\xi^{\prime}}{h} \\
& B:=R_{1 \overline{1} \bar{i} \bar{i}}=\frac{1}{(r f)^{2}}\left[r h-(1-\xi) \int_{0}^{r} h d s\right], i \geq 2 ; \\
& C:=R_{i \bar{i} i \bar{i}}=2 R_{i \bar{i} j \bar{j}}=\frac{2}{r^{2} f^{2}}\left(\int_{0}^{r} h d s-r h\right), i \neq j ; i, j \geq 2 .
\end{aligned}
$$

All other components are zero, except those obtained by the symmetric properties of $R$.

3. Characterizations of $\mathbf{B}^{\perp} \geq 0$ and $\mathbf{Q B} \geq 0$. In this section, we will give some characterizations of a $U(n)$-invariant Kähler metric to satisfy $\mathbf{B}^{\perp} \geq 0$ and QB $\geq 0$.

TheOREm 3.1. A $U(n)$-invariant Kähler metric on $\mathbb{C}^{n}$ satisfies $\mathbf{B}^{\perp} \geq 0$ if and only if $A+C \geq 0, B \geq 0$ and $C \geq 0$.

Proof. Since the metric is invariant under the $U(n)$ action, one just need to check the sign of the curvature at the point $p=\left(z_{1}, 0, \cdots, 0\right)$. Let $e_{i}$ be the unitary frame of $p$ as in (2.2). We shall compute $R(X, \bar{X}, Y, \bar{Y})$ for any given unitary pairs $X, Y \in T_{p}^{(1,0)}(M)$ first. As in [10], by applying a $U(n)$ action which preserves the point $p$, we may assume that

$$
X=x_{1} e_{1}+x_{2} e_{2}, Y=y_{1} e_{1}+y_{2} e_{2}+y_{3} e_{3}
$$

Then

$$
\begin{aligned}
R(X, \bar{X}, Y, \bar{Y})= & A\left|x_{1}\right|^{2}\left|y_{1}\right|^{2}+B\left(\left|x_{1}\right|^{2}\left|y_{2}\right|^{2}+\left|x_{1}\right|^{2}\left|y_{3}\right|^{2}+\left|x_{2}\right|^{2}\left|y_{1}\right|^{2}\right. \\
& \left.+x_{2} \overline{x_{1}} y_{1} \overline{y_{2}}+x_{1} \overline{x_{2}} y_{2} \overline{y_{1}}\right)+C\left(\left|x_{2}\right|^{2}\left|y_{2}\right|^{2}+\frac{1}{2}\left|x_{2}\right|^{2}\left|y_{3}\right|^{2}\right) .
\end{aligned}
$$

Since $X \perp Y$ which implies that $x_{1} \overline{y_{1}}+x_{2} \overline{y_{2}}=0$, we have 


$$
\begin{aligned}
R(X, \bar{X}, Y, \bar{Y})= & (A+C)\left|x_{1}\right|^{2}\left|y_{1}\right|^{2}+\frac{C}{2}\left|x_{2}\right|^{2}\left|y_{3}\right|^{2} \\
& +B\left(\left|x_{1}\right|^{2}\left|y_{2}\right|^{2}+\left|x_{2}\right|^{2}\left|y_{1}\right|^{2}-2\left|x_{1}\right|^{2}\left|y_{1}\right|^{2}+\left|x_{1}\right|^{2}\left|y_{3}\right|^{2}\right)
\end{aligned}
$$

Suppose $A+C \geq 0, B \geq 0$ and $C \geq 0$. Denote

$$
\alpha=\left|x_{1}\right|^{2}\left|y_{2}\right|^{2}+\left|x_{2}\right|^{2}\left|y_{1}\right|^{2}-2\left|x_{1}\right|^{2}\left|y_{1}\right|^{2} .
$$

To prove that $\mathbf{B}^{\perp} \geq 0$, it is sufficient to show that $\alpha \geq 0$.

If $y_{1} \neq 0$, then

$$
\left|y_{1}\right|^{2} \alpha=\left|x_{2}\right|^{2}\left(\left|y_{2}\right|^{2}-\left|y_{1}\right|^{2}\right)^{2} \geq 0
$$

because $x_{1} \overline{y_{1}}+x_{2} \overline{y_{2}}=0$. Hence $\alpha \geq 0$, and the coefficient of $B$ is non-negative. Similarly, if $y_{2} \neq 0$, then

$$
\left|y_{2}\right|^{2} \alpha=\left|x_{1}\right|^{2}\left(\left|y_{2}\right|^{2}-\left|y_{1}\right|^{2}\right)^{2} \geq 0
$$

and the coefficient of $B$ is also non-negative. If $y_{1}=y_{2}=0$, then the coefficient of $B$ is just $\left|x_{1}\right|^{2}\left|y_{3}\right|^{2}$ which is nonnegative. Thus, we conclude that if $A+C \geq 0, B \geq 0$ and $C \geq 0$ then $\mathbf{B}^{\perp} \geq 0$.

Conversely, suppose $\mathbf{B}^{\perp} \geq 0$, then $B \geq 0$ and $C \geq 0$ since $B=R_{1 \overline{1} i \bar{i}}$ and $C=2 R_{i \bar{i} j \bar{j}}$ for any $2 \leq i \neq j \leq n$. Take $x_{1}=y_{1}=y_{2}=-x_{2}=\frac{1}{\sqrt{2}}$ and $y_{3}=0$ which implies that $X, Y$ form a unitary pair. Then we get $A+C \geq 0$.

Next we want to characterize $U(n)$-invariant Kähler metric with $\mathbf{Q B} \geq 0$ in terms of $A, B, C$. By [2], a Kähler manifold $M$ satisfies $\mathbf{Q B} \geq 0$ if and only if the symmetric form $G-F$ is positive semi-definite on the space $\Omega_{\mathbb{R}}^{1,1}(M)$ of real $(1,1)$ forms at any $p \in M$, where

$$
F(\rho, \sigma)=\sum_{i, j, k, l} R_{i \bar{j} k \bar{l}} \rho^{i \bar{l}} \sigma^{k \bar{j}}=\sum_{i, j, k, l} R_{i \bar{l} k \bar{j}} \rho^{i \bar{l}} \sigma^{k \bar{j}}
$$

and

$$
G(\rho, \sigma)=\frac{1}{2}\left(R_{i \bar{j}} g_{k \bar{l}}+R_{k \bar{l}} g_{i \bar{j}}\right) \rho^{i \bar{l}} \sigma^{k \bar{j}}
$$

Here $\rho^{i \bar{l}}, \sigma^{k \bar{j}}$ are the local components of $\rho, \sigma$ with indices raised.

TheOrem 3.2. A $U(n)$-invariant Kähler metric satisfies $\mathbf{Q B} \geq 0$ if and only if $B \geq 0, A+(n-2) B+\frac{n}{2} C \geq 0$ and $B+\frac{n-1}{2} C \geq 0$.

Proof. As before, it is sufficient to prove that the theorem is true at the point $p=\left(z_{1}, 0, \cdots, 0\right)$. Now in the unitary frame $\left\{\frac{1}{\sqrt{h}} \partial_{z_{1}}, \frac{1}{\sqrt{f}} \partial_{z_{2}}, \cdots, \frac{1}{\sqrt{f}} \partial_{z_{n}}\right\}$ at the point $p=\left(z_{1}, 0, \cdots, 0\right)$, the Ricci curvature is diagonalized with

$$
\left\{\begin{array}{l}
R_{1 \overline{1}}=A+(n-1) B \\
R_{i \bar{i}}=B+C+\frac{n-2}{2} C=B+\frac{n}{2} C, \text { if } i \geq 2
\end{array}\right.
$$


So

$$
\begin{gathered}
G(\rho, \sigma)=\sum_{i, k} R_{i \bar{i}} \rho^{i \bar{k}} \sigma^{k \bar{i}} . \\
F(\rho, \sigma)=\sum_{i, j} R_{i \bar{i} j \bar{j}} \rho^{i \bar{i}} \sigma^{j \bar{j}}+\sum_{i \neq l, o r k \neq j} R_{i \bar{l} k \bar{j}} \rho^{i \bar{l}} \sigma^{k \bar{j}} \\
=\sum_{i, j} R_{i \bar{i} \bar{j} \bar{j}} \rho^{i \bar{i}} \sigma^{j \bar{j}}+\sum_{i \neq k} R_{i \bar{k} k \bar{i}} \rho^{i \bar{k}} \sigma^{k \bar{i}} \\
=\sum_{i, j} R_{i \bar{i} j \bar{j}} \rho^{i \bar{i}} \sigma^{j \bar{j}}+\sum_{i \neq j} R_{i \bar{i} j \bar{j}} \rho^{i \bar{j}} \sigma^{j \bar{i}}
\end{gathered}
$$

with $\rho^{i \bar{j}}=\overline{\rho^{j i}}=\rho_{i \bar{j}}$. Note that $a_{i}:=\rho_{i \bar{i}}$ is real. Then

$$
\begin{aligned}
& G(\rho, \rho)-F(\rho, \rho) \\
& =(A+(n-1) B) \sum_{k \geq 1}\left|\rho_{1 \bar{k}}\right|^{2}+\left(B+\frac{n}{2} C\right) \sum_{i \geq 2, k \geq 1}\left|\rho_{i \bar{k}}\right|^{2} \\
& -A a_{1}^{2}-2 B a_{1} \sum_{i \geq 2} a_{i}-C \sum_{i \geq 2} a_{i}^{2}-\frac{C}{2} \sum_{2 \leq i \neq j \leq n} a_{i} a_{j} \\
& -2 B \sum_{i \geq 2}\left|\rho_{1 \bar{i}}\right|^{2}-\frac{C}{2} \sum_{2 \leq i \neq j \leq n}\left|\rho_{i \bar{j}}\right|^{2} \\
& =(n-1) B a_{1}^{2}+\left(B+\left(\frac{n}{2}-1\right) C\right) \sum_{i \geq 2} a_{i}^{2}-2 B a_{1} \sum_{i \geq 2} a_{i} \\
& -\frac{C}{2} \sum_{2 \leq i \neq j \leq n} a_{i} a_{j}+\left(A+(n-2) B+\frac{n}{2} C\right) \sum_{i \geq 2}\left|\rho_{1 \bar{i}}\right|^{2} \\
& +\left(B+\left(\frac{n}{2}-\frac{1}{2}\right) C\right) \sum_{2 \leq i \neq j \leq n}\left|\rho_{i \bar{j}}\right|^{2} \\
& =B\left((n-1) a_{1}^{2}+\sum_{i \geq 2} a_{i}^{2}-2 a_{1} \sum_{i \geq 2} a_{i}\right)+C\left(\left(\frac{n}{2}-1\right) \sum_{i \geq 2} a_{i}^{2}-\frac{1}{2} \sum_{2 \leq i \neq j \leq n} a_{i} a_{j}\right) \\
& +\left(A+(n-2) B+\frac{n}{2} C\right) \sum_{i \geq 2}\left|\rho_{1 \bar{i}}\right|^{2}+\left(B+\left(\frac{n}{2}-\frac{1}{2}\right) C\right) \sum_{2 \leq i \neq j \leq n}\left|\rho_{i \bar{j}}\right|^{2} \\
& =B\left(\sum_{i \geq 2}\left(a_{1}-a_{i}\right)^{2}\right)+\frac{1}{2} C\left(\sum_{2 \leq i<j \leq n}\left(a_{i}-a_{j}\right)^{2}\right) \\
& +\left(A+(n-2) B+\frac{n}{2} C\right) \sum_{i \geq 2}\left|\rho_{1 \bar{i}}\right|^{2}+\left(B+\frac{n-1}{2} C\right) \sum_{2 \leq i \neq j \leq n}\left|\rho_{i \bar{j}}\right|^{2} .
\end{aligned}
$$


Denote $a_{1}-a_{i}$ by $x_{i}, i \geq 2$. Then

$$
\begin{aligned}
& G(\rho, \rho)-F(\rho, \rho) \\
= & B \sum_{i \geq 2} x_{i}^{2}+\frac{1}{2} C\left(\sum_{2 \leq i<j \leq n}\left(x_{i}-x_{j}\right)^{2}\right) \\
& +\left(A+(n-2) B+\frac{n}{2} C\right) \sum_{i \geq 2}\left|\rho_{1 \bar{i}}\right|^{2}+\left(B+\frac{n-1}{2} C\right) \sum_{2 \leq i \neq j \leq n}\left|\rho_{i \bar{j}}\right|^{2} \\
= & \frac{1}{n-1} B\left((n-1) \sum_{i \geq 2} x_{i}^{2}-\sum_{2 \leq i<j \leq n}\left(x_{i}-x_{j}\right)^{2}\right) \\
& +\frac{1}{n-1}\left(B+\frac{n-1}{2} C\right)\left(\sum_{2 \leq i<j \leq n}\left(x_{i}-x_{j}\right)^{2}\right) \\
& +\left(A+(n-2) B+\frac{n}{2} C\right) \sum_{i \geq 2}\left|\rho_{1 \bar{i}}\right|^{2}+\left(B+\frac{n-1}{2} C\right) \sum_{2 \leq i \neq j \leq n}\left|\rho_{i \bar{j}}\right|^{2} .
\end{aligned}
$$

Hence if $B \geq 0, A+(n-2) B+\frac{n}{2} C \geq 0$ and $B+\frac{n-1}{2} C \geq 0$, then $\mathbf{Q B} \geq 0$.

If we choose suitable $\rho_{i \bar{j}}$, we can conclude that $\mathbf{Q B} \geq 0$ then $B \geq 0, A+(n-$ 2) $B+\frac{n}{2} C \geq 0$ and $B+\frac{n-1}{2} C \geq 0$. This is also a consequence of Proposition 3.1 in the following.

To get a better understanding of the conditions in the more general setting, we have the following proposition. The first part is due to Niu [7], and the second part is an immediate consequence of the definition of $\mathbf{Q B} \geq 0$.

Proposition 3.1. Let $\left(M^{n}, g\right)$ be a Kähler manifold with complex dimension $n$. Suppose $\mathbf{Q B} \geq 0$ at a point $p$. Then for any unitary frame $e_{1}, \ldots, e_{n}$ at $p$, we have

(i) $R_{i \bar{i}}+R_{j \bar{j}}-2 R_{i \bar{i} j \bar{j}} \geq 0$ for all $i \neq j$; and

(ii) $R_{i \bar{i}}-R_{i \bar{i} i \bar{i}} \geq 0$.

Before we prove the proposition, let us consider the $U(n)$-invariant Kähler metric. With the above notations:

$$
\begin{cases}R_{1 \overline{1}}+R_{j \bar{j}}-2 R_{1 \overline{1} j \bar{j}}=A+(n-2) B+\frac{n}{2} C, & \text { for } j \geq 2 ; \\ R_{i \bar{i}}+R_{j \bar{j}}-2 R_{i \bar{i} j \bar{j}}=2\left(B+\frac{n-1}{2} C\right), & \text { for } 2 \leq i \neq j \\ R_{1 \overline{1}}-R_{1 \overline{1} 1 \overline{1}}=(n-1) B ; & \text { for } i \geq 2 .\end{cases}
$$

Hence the necessity part of Theorem 3.2 follows from the proposition. Hence the theorem means that for $U(n)$-invariant metric, the conditions (i) and (ii) are also sufficient for $\mathbf{Q B} \geq 0$.

Proof. [Proof of the Proposition] Suppose QB $\geq 0$. In a unitary frame,

$$
F(\rho, \rho)-G(\rho, \rho)=\sum_{i, j} R_{i \bar{i} \bar{j} \bar{j}} \rho_{i \bar{i}} \rho_{j \bar{j}}+\sum_{i \neq l, \text { or } k \neq j} R_{i \bar{l} k \bar{j}} \rho_{l \bar{i}} \rho_{j \bar{k}}-\sum_{i, k} R_{i \bar{i}} \rho_{k \bar{i}} \rho_{i \bar{k}} .
$$

where $\bar{\rho}_{i \bar{j}}=\rho_{j \bar{i}}$. Let $\rho_{i \bar{j}}=0$ for all $i, j$ except $\rho_{1 \overline{2}}$ and $\rho_{2 \overline{1}}$. Then

$$
\begin{aligned}
0 & \geq F(\rho, \rho)-G(\rho, \rho) \\
& =\left(2 R_{1 \overline{1} 2 \overline{2}}-R_{1 \overline{1}}-R_{2 \overline{2}}\right)\left|\rho_{1 \overline{2}}\right|^{2}+R_{2 \overline{1} 2 \overline{1}} \rho_{1 \overline{2}}^{2}+R_{1 \overline{2} 1 \overline{2}} \rho_{2 \overline{1}}^{2} \\
& =\left(2 R_{1 \overline{1} 2 \overline{2}}-R_{1 \overline{1}}-R_{2 \overline{2}}\right)\left|\rho_{1 \overline{2}}\right|^{2}+2 \Re\left(R_{2 \overline{1} 2 \overline{1}} \rho_{1 \overline{2}}^{2}\right) .
\end{aligned}
$$


Replace $\rho_{1 \overline{2}}$ by $\sqrt{-1} \rho_{1 \overline{2}}$, then we have

$$
\left(2 R_{1 \overline{1} 2 \overline{2}}-R_{1 \overline{1}}-R_{2 \overline{2}}\right)\left|\rho_{1 \overline{2}}\right|^{2}-2 \Re\left(R_{2 \overline{1} 2 \overline{1}} \rho_{1 \overline{2}}^{2}\right) \leq 0 .
$$

Hence we have

$$
R_{1 \overline{1}}+R_{2 \overline{2}}-2 R_{1 \overline{1} 2 \overline{2}} \geq 0
$$

This proves (i).

Now take $\rho_{1 \overline{1}} \neq 0$, and all other $\rho_{i \bar{j}}$ are zeros, we have

$$
0 \geq F(\rho, \rho)-G(\rho, \rho)=\left(R_{1 \overline{1} 1 \overline{1}}-R_{1 \overline{1}}\right)\left|\rho_{1 \overline{1}}\right|^{2} .
$$

From this (ii) follows.

4. $U(n)$-invariant metric with $\mathbf{Q B} \geq 0$. In this section, we will perturb a complete $U(n)$-invariant Kähler metric with positive bisectional curvature to obtain a $U(n)$-invariant Kähler metric which satisfies $\mathbf{Q B} \geq 0$ and does not satisfy $\mathbf{B}^{\perp} \geq 0$.

As mentioned in section 2, a complete $U(n)$-invariant Kähler metric with positive bisectional curvature is determined up to scaling by a smooth function $\xi$ on $[0, \infty)$ with $\xi(0)=0, \xi^{\prime}>0$ and $0<\xi<1$. We normalize the metric so that

$$
h(r)=\exp \left(-\int_{0}^{r} \frac{\xi(t)}{t} d t\right) .
$$

Fix a smooth cutoff function $\phi$ on $\mathbb{R}$ such that

(i) $0 \leq \phi \leq c_{0}$;

(ii) $\operatorname{supp} \phi \subset[-1,1]$

(iii) $\phi^{\prime}(0)=1$ and $\left|\phi^{\prime}\right| \leq 1$.

We have the following:

Theorem 4.1. For $n \geq 3$, for any $r_{0}>0$ there is $R>r_{0}$ and $\beta>0$ such that $\bar{\xi}(r)=\xi(r)-\beta \phi(r-R)$ will give a complete $U(n)$-invariant Kähler metric which satisfies $\mathbf{Q B} \geq 0$, and does not satisfy $\mathbf{B}^{\perp} \geq 0$ or Ric $\geq 0$.

Let $a=\lim _{r \rightarrow \infty} \xi(r)$. Then $0<a \leq 1$. By Theorem 2.1, if $\beta$ is chosen so that $\beta c_{0}<a$ and $R$ is chosen large enough, then $\bar{\xi}$ will give a complete $U(n)$-invariant Kähler metric on $\mathbb{C}^{n}$.

Let

$$
\begin{aligned}
\bar{h}(r) & =\exp \left(-\int_{0}^{r} \frac{\bar{\xi}(t)}{t} d t\right) ; \\
r \bar{f}(r) & =\int_{0}^{r} \bar{h} d s .
\end{aligned}
$$

In the unitary frame $\left\{\frac{1}{\sqrt{h}} \partial_{z_{1}}, \frac{1}{\sqrt{\bar{f}}} \partial_{z_{2}}, \cdots, \frac{1}{\sqrt{f}} \partial_{z_{n}}\right\}$ at a point $z=\left(z_{1}, 0, \cdots, 0\right)$, the curvature tensor of the perturbed metric are given by

$$
\begin{aligned}
& \bar{A}=\bar{R}_{1 \overline{1} 1 \bar{i}}=\frac{\bar{\xi}^{\prime}}{\bar{h}} ; \\
& \bar{B}=\bar{R}_{1 \overline{1} i \bar{i}}=\frac{1}{(r \bar{f})^{2}}\left[r \bar{h}-(1-\bar{\xi}) \int_{0}^{r} \bar{h}\right], i \geq 2 \\
& \bar{C}=\bar{R}_{i \bar{i} i \bar{i}}=2 \bar{R}_{i \bar{i} j \bar{j}}=\frac{2}{r^{2} \bar{f}^{2}}\left(\int_{0}^{r} \bar{h} d s-r \bar{h}\right), i \neq j, i, j \geq 2 .
\end{aligned}
$$


In order to prove the theorem, we need to obtain some estimates, which are rather elementary and some of them may already be obtained in [10].

Lemma 4.1. Let $\xi$ be as above. Suppose $\lim _{r \rightarrow \infty} \xi=a, 0<a \leq 1$. We have the following:

(i) $\lim _{r \rightarrow \infty} h(r)=0$, and for any $r_{0}, \lim _{r \rightarrow \infty} \frac{h\left(r+r_{0}\right)}{h(r)}=1$.

(ii) $\lim _{r \rightarrow \infty} \int_{0}^{r} h=\infty$ and for any $r_{0}$,

$$
\lim _{r \rightarrow \infty} \frac{\int_{0}^{r+r_{0}} h}{\int_{0}^{r} h}=1 .
$$

(iii) $\left(r h+(\xi-1) \int_{0}^{r} h\right)^{\prime}>0$ for $r>0$.

(iv)

$$
\lim _{r \rightarrow \infty} \frac{r h}{\int_{0}^{r} h}=1-a .
$$

(v) For any $\epsilon>0$, and for any $r_{0}>0$, there is $R>r_{0}$ such that

$$
\xi^{\prime}(R)-\epsilon h(R) C(R)<0 .
$$

(vi) $\lim _{r \rightarrow \infty} h(r) C(r)=0$.

(vii) For all $\epsilon>0$, there exists $\delta>0$ such that if $R \geq 3, \delta \geq \eta \geq 0$ is a smooth function with support in $[R-1, R+1]$, then for all $r \geq 0$,

$$
h(r) \leq \bar{h}(r) \leq(1+\epsilon) h(r) ; \quad \text { and } \quad \int_{0}^{r} h \leq \int_{0}^{r} \bar{h} \leq(1+\epsilon) \int_{0}^{r} h
$$

where $\bar{h}(r)=\exp \left(-\int_{0}^{r} \frac{\bar{\xi}(t)}{t} d t\right)$ and $\bar{\xi}=\xi-\eta$.

Proof. (i) Since $\lim _{r \rightarrow \infty} \xi=a>0, \int_{1}^{r} \frac{\xi(t)}{t} d t \rightarrow \infty$ as $r \rightarrow \infty$. Hence $h(r) \rightarrow 0$ as $r \rightarrow \infty$. This proves the first assertion of (i). For fixed $r_{0}$ (which may be negative),

$$
\frac{h\left(r+r_{0}\right)}{h(r)}=\exp \left(-\int_{r}^{r+r_{0}} \frac{\xi(t)}{t} d t\right) .
$$

Since for fixed $r_{0}, \int_{r}^{r+r_{0}} \frac{\xi(t)}{t} d t \rightarrow 0$ as $r \rightarrow \infty$, the second assertion of (i) is also true.

(ii) Since $\xi \leq a \leq 1, h(r) \geq h(1) r^{-1}$ for $r \geq 1$. Hence $\int_{0}^{r} h \rightarrow \infty$ as $r \rightarrow \infty$.

$$
\frac{\int_{0}^{r+r_{0}} h}{\int_{0}^{r} h}=1+\frac{\int_{r}^{r+r_{0}} h}{\int_{0}^{r} h} .
$$

Since $h^{\prime}<0$, and $h(0)=1, h \leq 1$. Since $\int_{0}^{r} h \rightarrow \infty$ as $r \rightarrow \infty$, we have

$$
\lim _{r \rightarrow \infty} \frac{\int_{0}^{r+r_{0}} h}{\int_{0}^{r} h}=1
$$

(iii)

$$
\left(r h+(\xi-1) \int_{0}^{r} h\right)^{\prime}=r h^{\prime}+h+\xi^{\prime} \int_{0}^{r} h+(\xi-1) h=\xi^{\prime} \int_{0}^{r} h>0
$$


for $r>0$, because $\xi=-\frac{r h^{\prime}}{h}>0$.

(iv) By (ii) and the l'Hospital rule, we have

$$
\lim _{r \rightarrow \infty} \frac{r h}{\int_{0}^{r} h}=\lim _{r \rightarrow \infty} \frac{r h^{\prime}+h}{h}=1-a .
$$

(v) Suppose (v) is not true. Then there is $r_{0}>0$ and $\epsilon>0$ such that $\xi^{\prime}(r) \geq$ $\epsilon h(r) C(r)$ for all $r \geq r_{0}$. By (iv)

$$
\lim _{r \rightarrow \infty} C(r) \int_{0}^{r} h=\lim _{r \rightarrow \infty} 2\left(1-\frac{r h}{\int_{0}^{r} h}\right)=2 a>0 .
$$

Hence there exists $r_{1}>r_{0}$ such that for all $r \geq r_{1}$, we have

$$
C(r) \geq \frac{a}{\int_{0}^{r} h}
$$

Then for all $r \geq r_{1}$, we have

$$
\xi^{\prime}(r) \geq a \epsilon \frac{h}{\int_{0}^{r} h} .
$$

Integrate the above inequality, we have

$$
\xi(r)-\xi\left(r_{1}\right) \geq a \epsilon \log \left(\frac{\int_{0}^{r} h}{\int_{0}^{r_{1}} h}\right) .
$$

This is impossible because $\xi$ is bounded and $\int_{0}^{r} h \rightarrow \infty$ as $r \rightarrow \infty$.

(vi)

$$
h(r) C(r)=\frac{2 h(r)}{\int_{0}^{r} h}\left(1-\frac{r h}{\int_{0}^{r} h}\right) \leq \frac{2}{r} \frac{r h}{\int_{0}^{r} h} .
$$

By (iv), we conclude that $h(r) C(r) \rightarrow 0$ as $r \rightarrow \infty$.

(vii) It is sufficient to show the first part.

$$
\bar{h}(r)=h(r) e^{\int_{0}^{r} \frac{\eta(t)}{t} d t} \leq h(r) e^{\int_{R-1}^{R+1} \frac{\eta(t)}{t} d t} \leq h(r) e^{2 \delta \frac{1}{R-1}} \leq h(r) e^{\delta} .
$$

We take $\delta=\ln (1+\epsilon)$, then we have $\bar{h}(r) \leq(1+\epsilon) h(r)$. Since $\eta \geq 0, h(r) \leq \bar{h}(r)$.

LEMMA 4.2. There is $2 \geq \alpha>0$ such that for any $r_{0}>0$ there is $R>r_{0}$ satisfying the following: If $\bar{\xi}(r)=\xi(r)-\alpha h(R) C(R) \phi(r-R)$, then $\bar{\xi}$ determines a complete $U(n)$-invariant Kähler metric on $\mathbb{C}^{n}$ such that

(i) $\bar{A}(R)+\bar{C}(R)<0$;

(ii) $\bar{A}+\frac{n}{2} \bar{C}>0$ on $[R-1, R+1]$; and

(iii) $\bar{A}(R)+(n-1) \bar{B}(R)<0$.

Proof. Let $\epsilon>0$ and $R \geq 3$ to be chosen later. Then by Lemma 4.1(vii), there is $a>\delta>0$ independent of $R$, such that if $\bar{\xi}(r)=\xi(r)-\beta \phi(r-R)$ with $\beta>0$ and $\beta c_{0}<\delta$, then $\bar{\xi}$ determines a complete $U(n)$-invariant Kähler metric on $\mathbb{C}^{n}$ such that for all $r$

$$
h(r) \leq \bar{h}(r) \leq(1+\epsilon) h(r) ; \int_{0}^{r} h \leq \int_{0}^{r} \bar{h} \leq(1+\epsilon) \int_{0}^{r} \bar{h} .
$$


Then for any $r_{0}>3$, there is $r_{1}>r_{0}$ which is independent of $R$ such that if $r>r_{1}$, then

$$
\begin{aligned}
\bar{C}(r) & =\frac{2}{\int_{0}^{r} \bar{h}}\left(1-\frac{r \bar{h}}{\int_{0}^{r} \bar{h}}\right) \\
& \leq \frac{2}{\int_{0}^{r} \bar{h}}\left(1-\frac{r h}{(1+\epsilon) \int_{0}^{r} h}\right) \\
& \leq \frac{2}{\int_{0}^{r} \bar{h}}\left(1-\frac{1-a-\epsilon}{1+\epsilon}\right) \\
& \leq \frac{2}{\int_{0}^{r} h} \frac{a+2 \epsilon}{1+\epsilon}
\end{aligned}
$$

where we have used Lemma 4.1(iv). On the other hand, by Lemma 4.1(iv), we may choose $r_{1}$ large enough so that if $r \geq r_{1}$, then

$$
C(r)=\frac{2}{\int_{0}^{r} h}\left(1-\frac{r h}{\int_{0}^{r} h}\right) \geq \frac{2}{\int_{0}^{r} h}(a-\epsilon) .
$$

Hence if $r>r_{1}$, and $\epsilon<a$, then

$$
\bar{C}(r) \leq \frac{a+2 \epsilon}{(a-\epsilon)(1+\epsilon)} C(r) .
$$

Suppose $R>r_{1}$, then

$$
\begin{aligned}
\bar{A}(R)+\bar{C}(R) & =\frac{\xi^{\prime}(R)-\beta}{\bar{h}(R)}+\bar{C}(R) \\
& \leq \frac{\xi^{\prime}(R)-\beta}{\bar{h}(R)}+\frac{a+2 \epsilon}{(a-\epsilon)(1+\epsilon)} C(R) \\
& \leq \frac{\xi^{\prime}(R)-\beta}{(1+\epsilon) h(R)}+\frac{a+2 \epsilon}{(a-\epsilon)(1+\epsilon)} C(R) \\
& =\frac{1}{(1+\epsilon) h(R)}\left(\xi^{\prime}(R)-\beta+\frac{a+2 \epsilon}{a-\epsilon} h(R) C(R)\right)
\end{aligned}
$$

provided that $\xi^{\prime}(R)-\beta<0$. On the other hand, if $R>r_{1}$ as above, by Lemma 4.1(i) and (ii) we may choose a larger $r_{1}$ so that for $r \in[R-1, R+1]$, we have

$$
\begin{aligned}
\bar{C}(r) & =\frac{2}{\int_{0}^{r} \bar{h}}\left(1-\frac{r \bar{h}}{\int_{0}^{r} \bar{h}}\right) \\
& \geq \frac{2}{\int_{0}^{r} \bar{h}}\left(1-\frac{(1+\epsilon) r h}{\int_{0}^{r} h}\right) \\
& \geq \frac{2}{\int_{0}^{r} \bar{h}}\left(a-2 \epsilon+a \epsilon-\epsilon^{2}\right) \\
& \geq \frac{2}{(1+\epsilon) \int_{0}^{r} h}\left(a-2 \epsilon+a \epsilon-\epsilon^{2}\right) \\
& \geq \frac{2}{(1+\epsilon)^{2} \int_{0}^{R} h}\left(a-2 \epsilon+a \epsilon-\epsilon^{2}\right)
\end{aligned}
$$


provided $a-2 \epsilon+a \epsilon-\epsilon^{2}>0$. We choose $\epsilon>0$ so that it satisfies this condition.

On the other hand,

$$
C(R)=\frac{2}{\int_{0}^{R} h}\left(1-\frac{R h}{\int_{0}^{R} h}\right) \leq \frac{2}{\int_{0}^{R} h}(a+\epsilon)
$$

if $r_{1}$ is large enough depending on $\epsilon$ and $R>r_{1}$ by Lemma 4.1(iv). Hence if $\epsilon$ and $r_{1}$ satisfy the above conditions, then for $r \in[R-1, R+1]$

$$
\bar{C}(r) \geq \frac{a-2 \epsilon+a \epsilon-\epsilon^{2}}{(a+\epsilon)(1+\epsilon)^{2}} C(R) .
$$

Therefore if $\epsilon>0$ satisfies $a>\epsilon$ and $a-2 \epsilon+a \epsilon-\epsilon^{2}>0$, then we can find $r_{1}>r_{0}$ such that if $R>r_{1}$, then for $r \in[R-1, R+1]$,

$$
\begin{aligned}
\bar{A}(r)+\frac{n}{2} \bar{C}(r) & \geq \frac{\xi^{\prime}(r)-\beta}{\bar{h}(r)}+\frac{n}{2} \bar{C}(r) \\
& \geq \frac{-\beta}{h(r)}+\frac{n}{2} \frac{a-2 \epsilon+a \epsilon-\epsilon^{2}}{(a+\epsilon)(1+\epsilon)^{2}} C(R) \\
& \geq \frac{-\beta}{(1-\epsilon) h(R)}+\frac{n}{2} \frac{a-2 \epsilon+a \epsilon-\epsilon^{2}}{(a+\epsilon)(1+\epsilon)^{2}} C(R) \\
& =\frac{1}{(1-\epsilon) h(R)}\left[-\beta+\frac{n}{2}(1-\epsilon) \frac{a-2 \epsilon+a \epsilon-\epsilon^{2}}{(a+\epsilon)(1+\epsilon)^{2}} h(R) C(R)\right] .
\end{aligned}
$$

Choose $\epsilon>0$ which also satisfies:

$$
\frac{n}{2}(1-\epsilon) \frac{a-2 \epsilon+a \epsilon-\epsilon^{2}}{(a+\epsilon)(1+\epsilon)^{2}}>\frac{a+2 \epsilon}{a-\epsilon} .
$$

This can be done because $n \geq 3$. Let $\beta=\alpha h(R) C(R)$, where $2 \geq \alpha>0$ is a fixed constant depending on $\epsilon, a$ and $n$ such that

$$
\frac{n}{2}(1-\epsilon) \frac{a-2 \epsilon+a \epsilon-\epsilon^{2}}{(a+\epsilon)(1+\epsilon)^{2}}>\alpha>\frac{a+2 \epsilon}{a-\epsilon} .
$$

We can also choose $R$ large enough, so that $\beta c_{0}<\delta$ by Lemma 4.1(vi). Hence by Lemma 4.1(v), (4.5) and (4.7), one can find $R>r_{0}$ which is large enough, so that

$$
\bar{A}(R)+\bar{C}(R)<0
$$

and

$$
\bar{A}(r)+\frac{n}{2} \bar{C}(r)>0
$$

for $r \in[R-1, R+1]$. Hence (i) and (ii) are satisfied. It remains to show that one can choose $R$ so that (iii) is also satisfies. First note that

$$
\begin{aligned}
\bar{B}(R) & =\frac{1}{\int_{0}^{R} \bar{h}}\left(\frac{R \bar{h}(R)}{\int_{0}^{R} \bar{h}}-(1-\xi(R)+\alpha h(R) C(R) \phi(0))\right) \\
& \leq \frac{1}{\int_{0}^{R} \bar{h}}\left((1+\epsilon) \cdot \frac{R h(R)}{\int_{0}^{R} h}-(1-\xi(R)+\alpha h(R) C(R) \phi(0))\right) .
\end{aligned}
$$


By Lemma 4.1(iv), given $\epsilon_{1}>0$ one can choose $R$ large enough, so that

$$
\bar{B}(R) \leq \frac{\epsilon_{1}}{\int_{0}^{R} h} .
$$

On the other hand by (4.4) and (4.3)

$$
\bar{A}(R) \leq-\bar{C}(R) \leq-\frac{2(a-\epsilon)}{\int_{0}^{R} h} .
$$

Hence

$$
\bar{A}(R)+(n-1) \bar{B}(R)<0
$$

if $R$ is chosen large enough. Hence (iii) is also satisfied.

Lemma 4.3. Let $\phi$ as before and let $\bar{\xi}(r)=\xi(r)-\beta \phi(r-R)$ with $\beta>0$ so that $\bar{\xi}(r)>0$.

(i) Suppose $\beta c_{0} \leq c_{1}$ and $\beta(R) \int_{0}^{R} h \rightarrow 0$ as $R \rightarrow+\infty$. Then there exists $r_{0}>0$ such that if $R>r_{0}$, then $\bar{B}>0$ on $[0, \infty)$.

(ii) There exists $\delta>0$ such that if $\beta c_{0}<\delta$, then there exists $r_{0}>0$ such that if $R>r_{0}$, then $\bar{C}>0$ on $[0, \infty)$.

Proof. Denote $\beta \phi(r-R)$ by $\eta(r)$. To prove (i), suppose $\beta c_{0} \leq c_{1}$. Then as in the proof of Lemma 4.1(vii), there is a constant $c_{2}$ depending only on $c_{1}$ such that if $r \geq 3$, then

$$
h(r) \leq \bar{h}(r) \leq\left(1+c_{2}\right) h(r), \quad \int_{0}^{r} h \leq \int_{0}^{r} \bar{h} \leq\left(1+c_{2}\right) \int_{0}^{r} h .
$$

If $r<R-1$, then $\bar{B}(r)=B(r)>0$. If $R \geq 4$ and $r \geq R-1$, then

$$
\begin{aligned}
(r \bar{f})^{2} \bar{B}(r)= & r \bar{h}(r)-(1-\bar{\xi}(r)) \int_{0}^{r} \bar{h}(t) d t \\
= & \int_{0}^{r}\left[(t \bar{h}(t))^{\prime}-(1-\bar{\xi}(r)) \bar{h}(t)\right] d t \\
= & \int_{0}^{r}(\bar{\xi}(r)-\bar{\xi}(t)) \bar{h}(t) d t \\
= & \int_{0}^{r}(\xi(r)-\xi(t)) \bar{h}(t) d t-\int_{R-1}^{r}(\eta(r)-\eta(t)) \bar{h}(t) d t-\eta(r) \int_{0}^{R-1} h(t) d t \\
\geq & \int_{0}^{r}(\xi(r)-\xi(t)) h(t) d t-2 c_{1}\left(1+c_{2}\right) h(R-1) \\
& -\beta c_{0} \int_{0}^{R-1} h(t) d t \quad\left(\operatorname{since} \xi^{\prime}>0, h^{\prime}<0\right) \\
\geq & r h(r)+(\xi(r)-1) \int_{0}^{r} h(t) d t-2 c_{1}\left(1+c_{2}\right) h(R-1)-c_{0} \beta \int_{0}^{R} h(t) d t .
\end{aligned}
$$

By Lemma 4.1(iii), there is a constant $c_{3}>0$ such that

$$
r h(r)+(\xi(r)-1) \int_{0}^{r} h(t) d t \geq c_{3},
$$


for all $r \geq 1$. Hence if $R \geq 4$, we have

$$
(r \bar{f})^{2} \bar{B}(r) \geq c_{3}-2 c_{1}\left(1+c_{2}\right) h(R-1)-c_{0} \beta \int_{0}^{R} h(t) d t .
$$

By Lemma 4.1(i), we know that $h(R-1) \rightarrow 0$ as $R \rightarrow \infty$. From this it is easy to see that $\bar{B}>0$ if $R$ is large.

To prove (ii), let $\epsilon>0$ be such that $1-(1+\epsilon)(1-a+\epsilon)>0$. Such $\epsilon$ exists because $a>0$. By Lemma 4.1(vii) there exists $\delta>0$ such that if $\beta c_{0}<\delta$, then (4.3) is true for all $r$ if $R \geq 3$. If $r<R-1$ then $\bar{C}(r)=C(r)>0$. If $r \geq R-1$, then

$$
\begin{aligned}
r^{2} \bar{f}^{2} \bar{C} & =r \bar{f}-r \bar{h}(r) \\
& =\int_{0}^{r} \bar{h}(t) d t-r \bar{h}(r) \\
& \geq \int_{0}^{r} h-r \bar{h}(r) \\
& =\left(\int_{0}^{r} h\right)\left(1-\frac{r h(r)}{\int_{0}^{r} h} \frac{\bar{h}(r)}{h(r)}\right) \\
& \geq\left(\int_{0}^{r} h\right)\left(1-(1+\epsilon) \frac{r h(r)}{\int_{0}^{r} h}\right) \\
& \geq\left(\int_{0}^{r} h\right)(1-(1+\epsilon)(1-a+\epsilon)) \\
& >0
\end{aligned}
$$

if $R$ is large enough, where we have used Lemma 4.1(iv). This completes the proof of (ii).

Proof of Theorem 4.1. Let $\alpha$ be as in Lemma 4.2. For any $r_{0}>0$, let $R>r_{0}$ be such that Lemma 4.2 is true. One can choose $r_{0}$ large enough so that the conclusions of Lemma 4.3 are true for $\beta=\alpha h(R) C(R)$, because $h(r) C(r) \rightarrow 0, h(r) \rightarrow 0$ as $r \rightarrow \infty$ and $c(r) \sim \frac{1}{\int_{0}^{r} h}$. Since $\bar{A}(r)>0$ on $[0, R-1] \cup[R+1, \infty)$ and $\bar{B}(r), \bar{C}(r)>0$ for all $r$, the metric determined by $\bar{\xi}$ satisfies $\mathbf{Q B} \geq 0$ by Lemma 4.2(ii) and Theorem 3.2. Since $\bar{A}(R)+\bar{C}(R)<0$, it does not satisfies $\mathbf{B}^{\perp} \geq 0$. Since $\bar{A}(R)+(n-1) \bar{B}(R)<0$, it does not satisfy Ric $\geq 0$.

REMARK 4.1. Using similar method one may also construct, in a more simple way, complete $U(n)$-invariant Kähler metrics with $\mathbf{B}^{\perp} \geq 0$ which does not satisfy $\mathbf{B} \geq 0$ at some points.

5. Preservation of $\mathrm{QB} \geq 0$ in Kähler-Ricci flow. In this section, we will prove that $\mathbf{Q B} \geq 0$ is preserved under the Kähler-Ricci flow for complete $U(n)$ invariant metrics, provided the curvature is bounded. We will also prove that Ric $\geq 0$ is preserved with an additional assumption.

Let $g_{0}$ be a complete $U(n)$-invariant Kähler metric on $\mathbb{C}^{n}$, and $g(t)$ is a complete $U(n)$-invariant solution of the Kähler-Ricci flow on $\mathbb{C}^{n} \times[0, T], T>0$ with $g(0)=g_{0}$. We have a time-dependent orthonormal moving frame $\left\{e_{1}(t)=\frac{1}{\sqrt{h(r, t)}} \partial_{z_{1}}, e_{2}(t)=\right.$ 


$$
\begin{aligned}
& \left.\frac{1}{\sqrt{f(r, t)}} \partial_{z_{2}}, \cdots, e_{n}(t)=\frac{1}{\sqrt{f(r, t)}} \partial_{z_{n}}\right\} \text { at the point } z=\left(z_{1}, 0, \cdots, 0\right) . \text { Denote } \\
& \begin{aligned}
A(z, t) & =\operatorname{Rm}_{g(t)}\left(e_{1}(t), \bar{e}_{1}(t), e_{1}(t), \bar{e}_{1}(t)\right) \\
B(z, t) & =\operatorname{Rm}_{g(t)}\left(e_{1}(t), \bar{e}_{1}(t), e_{i}(t), \bar{e}_{i}(t)\right) \\
C(z, t) & =\operatorname{Rm}_{g(t)}\left(e_{i}(t), \bar{e}_{i}(t), e_{i}(t), \bar{e}_{i}(t)\right)=2 \operatorname{Rm}_{g(t)}\left(e_{i}(t), \bar{e}_{i}(t), e_{j}(t), \bar{e}_{j}(t)\right),
\end{aligned}
\end{aligned}
$$

where $2 \leq i \neq j \leq n$. Direct computations show [13] :

$$
\begin{aligned}
& \left(\frac{\partial}{\partial t}-\Delta\right) A=A^{2}+2(n-1) B^{2} \\
& \left(\frac{\partial}{\partial t}-\Delta\right) B=-B^{2}+A B+\frac{n}{2} B C, \\
& \left(\frac{\partial}{\partial t}-\Delta\right) C=2 B^{2}+\frac{n}{2} C^{2} .
\end{aligned}
$$

Theorem 5.1. Assume $g(t), t \in[0, T]$ is a complete solution of the $U(n)$ invariant Kähler-Ricci flow on $\mathbb{C}^{n}$, and $\operatorname{Rm}(z, t)$ is uniformly bounded on $\mathbb{C}^{n} \times[0, T]$. Suppose $g(0)$ satisfies $\mathbf{Q B} \geq 0$. Then $g(t)$ satisfies $\mathbf{Q B} \geq 0$ for all $t \in[0, T]$.

Proof. By Theorem 3.2, it is sufficient to prove that if $B \geq 0, E:=A+(n-$ 2) $B+\frac{n}{2} C \geq 0$ and $E:=B+\frac{n-1}{2} C \geq 0$ at $t=0$, then $B, E, F$ are also nonnegative for all $t \in[0, T]$. We have the following system for $B, E, F$

$$
\left\{\begin{array}{l}
\left(\frac{\partial}{\partial t}-\Delta\right) B=B\left(-B+A+\frac{n}{2} C\right) \\
\left(\frac{\partial}{\partial t}-\Delta\right) E=\left(A-\frac{n}{2} C\right) E+\frac{2 n(n-2)}{n-1} B F+\frac{n^{2}}{2} C^{2}+\frac{2 n}{n-1} B^{2} \\
\left(\frac{\partial}{\partial t}-\Delta\right) F=B E+\frac{n(n-1)}{4} C^{2} .
\end{array}\right.
$$

We may proceed as in [8, Chapter 3, section 8]. Since the curvature of $g(t)$ is bounded on $\mathbb{C}^{n} \times[0, T]$, by $[5$, Lemma 1.1], for all $a>0$ and $c>0$, there exists a smooth function $\phi$ and a positive constant $b$ such that

$$
e^{a\left(1+r_{o}(x)\right)} \leq \phi(x, t) \leq e^{b\left(1+r_{o}(x)\right)}
$$

and

$$
\left(\frac{\partial}{\partial t}-\Delta\right) \phi>c \phi
$$

on $\mathbb{C}^{n} \times[0, T]$, where $r_{o}(x)$ is the distance from a fixed point $o$ with respect to the initial metric $g(0)$. Let $\phi$ be such a function with $a=1$, and $c$ to be determined later. Let $\epsilon>0$ be any constant and let $B_{1}=B+\epsilon \phi, E_{1}=E+\epsilon \phi$, and $F_{1}=F+\epsilon \phi$. We want to prove that $B_{1}, E_{1}, F_{1}$ are nonnegative in $\mathbb{C}^{n} \times[0, T]$. Suppose not, since $B, E, F$ are bounded and they are nonnegative at $t=0$, we conclude that there exist $\left(x_{0}, t_{0}\right) \in \mathbb{C}^{n} \times(0, T]$ such that

$$
\min \left\{B_{1}\left(x_{0}, t_{0}\right), E_{1}\left(x_{0}, t_{0}\right), F_{1}\left(x_{0}, t_{0}\right)\right\}=0
$$

and $B_{1}, E_{1}, F_{1} \geq 0$ on $\mathbb{C}^{n} \times\left[0, t_{0}\right]$. 
Suppose $B_{1}\left(x_{0}, t_{0}\right)=0$, then at $\left(x_{0}, t_{0}\right)$

$$
\begin{aligned}
0 & \geq\left(\frac{\partial}{\partial t}-\Delta\right) B_{1} \\
& >B_{1}\left(-B+A+\frac{n}{2} C\right)+\epsilon \phi\left[c-\left(-B+A+\frac{n}{2} C\right)\right] \\
& =\epsilon \phi\left[c-\left(-B+A+\frac{n}{2} C\right)\right] .
\end{aligned}
$$

Similarly, suppose $E_{1}\left(x_{0}, t_{0}\right)=0$, then

$$
\begin{aligned}
0 & \geq\left(\frac{\partial}{\partial t}-\Delta\right) E_{1} \\
& >\left(A-\frac{n}{2} C\right) E+\frac{2 n(n-2)}{n-1} B F+c \epsilon \phi \\
& =\frac{2 n(n-2)}{n-1} B F+\epsilon \phi\left[c-\left(A-\frac{n}{2} C\right)\right] \\
& \geq\left\{\begin{array}{cl}
\epsilon \phi\left(c-\left(A-\frac{n}{2} C\right)\right), & \text { if } B\left(x_{0}, t_{0}\right) F\left(x_{0}, t_{0}\right) \geq 0 ; \\
\epsilon \phi\left(c-\left(A-\frac{n}{2} C\right)-\frac{2 n(n-2) B}{n-1}\right), & \text { if } B\left(x_{0}, t_{0}\right)>0 \text { and } F\left(x_{0}, t_{0}\right)<0 ; \\
\epsilon \phi\left(c-\left(A-\frac{n}{2} C\right)-\frac{2 n(n-2) F}{n-1}\right), & \text { if } B\left(x_{0}, t_{0}\right)<0 \text { and } F\left(x_{0}, t_{0}\right)>0 .
\end{array}\right.
\end{aligned}
$$

Here we have used the fact that $B_{1}\left(x_{0}, t_{0}\right) \geq 0$ and $F_{1}\left(x_{0}, t_{0}\right) \geq 0$.

Suppose $F_{1}\left(x_{0}, t_{0}\right)=0$, then

$$
\begin{aligned}
0 & \geq\left(\frac{\partial}{\partial t}-\Delta\right) F_{1} \\
& >B E+\epsilon c \phi \\
& \geq \begin{cases}\epsilon c \phi, & \text { if } B\left(x_{0}, t_{0}\right) E\left(x_{0}, t_{0}\right) \geq 0 ; \\
\epsilon \phi(c-B), & \text { if } B\left(x_{0}, t_{0}\right)>0 \text { and } E\left(x_{0}, t_{0}\right)<0 ; \\
\epsilon \phi(c-E), & \text { if } B\left(x_{0}, t_{0}\right)<0 \text { and } E\left(x_{0}, t_{0}\right)>0 .\end{cases}
\end{aligned}
$$

Here we have used the fact that $B_{1}\left(x_{0}, t_{0}\right) \geq 0$ and $E_{1}\left(x_{0}, t_{0}\right) \geq 0$.

By (5.3)-(5.5), if we choose $c$ such that

$$
c>\sup _{\mathbb{C}^{n} \times[0, T]}\left(\left|-B+A+\frac{n}{2} C\right|+\left|A-\frac{n}{2} C\right|+\frac{2 n(n-2)}{n-1}(|B|+|F|)+|E|\right),
$$

which is independent of $\epsilon$. Then we have a contradiction. Hence $B_{1}, E_{1}, F_{1}$ are nonnegative on $\mathbb{C}^{n} \times[0, T]$. Let $\epsilon \rightarrow 0$, we conclude the theorem is true.

TheOrem 5.2. Assume $g(t), t \in[0, T]$ is a complete solution of the $U(n)$ invariant Kähler-Ricci flow on $\mathbb{C}^{n}$, and $\operatorname{Rm}(z, t)$ is uniformly bounded on $\mathbb{C}^{n} \times[0, T]$. Suppose $g(0)$ satisfies Ric $\geq 0$ and $B \geq 0$. Then $g(t)$ satisfies Ric $\geq 0$ and $B \geq 0$ for all $t$.

Proof. By (3.1), it is sufficient to prove that if $B \geq 0, G:=A+(n-1) B \geq 0$ and $H:=B+\frac{n}{2} C \geq 0$ at $t=0$, then these are still true for all $t \in[0, T]$.

$$
\left\{\begin{array}{l}
\left(\frac{\partial}{\partial t}-\Delta\right) B=B\left(-B+A+\frac{n}{2} C\right) \\
\left(\frac{\partial}{\partial t}-\Delta\right) G=A G+(n-1) B H \\
\left(\frac{\partial}{\partial t}-\Delta\right) H=B G+\frac{n}{2} C H .
\end{array}\right.
$$


Then we can proceed as in the proof of the previous theorem.

REMARK 5.1. In the proofs of the above two theorems, we may prove that $B \geq 0$ is preserved first. Then the rest of the proofs are somewhat simpler.

\section{REFERENCES}

[1] A. Chau And L.-F. TAm, On quadratic orthogonal bisectional curvature, J. Differential Geom., $92(2012)$, pp. 187-200.

[2] A. Chau And L. F. TAm, Kähler C-spaces and Quadratic Bisectional curvature, J. Differential Geom., 94 (2013), pp. 409-468.

[3] S. I. Goldberg and S. Kobayashi, Holomorphic bisectional curvature, J. Differential Geom., 1 (1967), pp. 225-233.

[4] Q. Li, D. Wu, AND F. Zheng, An example of a compact Kähler manifold with nonnegative quadratic bisectional curvature, Proc. Amer. Math. Soc., 141:6 (2013), pp. 2117-2126.

[5] L. Ni And L.-F. TAM, Plurisubharmonic Functions and the Kähler-Ricci Flow. American Journal of Mathematics, 125 (2003), pp.623-654.

[6] L. Ni AND L.-F. TAM, Poincaré-Lelong equation via the Hodge Laplace heat equation, Compos. Math., 149:11 (2013), pp. 1856-1870.

[7] Y. NiU, A note on nonnegative quadratic orthogonal bisectional curvature, Proc. Amer. Math. Soc., 142:11 (2014), pp. 3975-3979.

[8] M. H. Protter, Maximum principles in differential equations, Prentice Hall (1967).

[9] D. Wu, S.-T. YAu, And F. Zheng, A degenerate Monge Ampère equation and the boundary classes of Kähler cones, Math. Res. Lett., 16:2 (2009), pp. 365-374.

[10] H. Wu and F. Zheng, Examples of Positively Curved Complete Kähler Manifolds, Geometry and analysis. No. 1, pp. 517-542, Adv. Lect. Math., 17, Int. Press, Somerville, MA, 2011.

[11] X. Zhang, On the boundary of Kähler cones, Proc. Amer. Math. Soc., 140:2 (2012), pp. 701705.

[12] F. Zheng, Complex differential geometry, American Mathematical Society, International Press, 2000.

[13] B. YAng And F. Zheng, U(n)-invariant Kähler-Ricci flow with non-negative curvature, Comm. Anal. Geom. , 21:2 (2013), 251-294. 